\title{
STARD1: a new rising StAR in cholesterol-mediated hepatocarcinogenesis
}

\author{
Salvatore Papa ${ }^{1}$, Concetta Bubici ${ }^{2}$, Wing-Kin Syn ${ }^{3,4,5}$ \\ ${ }^{1}$ Leeds Institute of Medical Research at St. James', Faculty of Medicine and Health, University of Leeds, St. James' University Hospital, Leeds, UK; \\ ${ }^{2}$ Centre for Genome Engineering and Maintenance, Department of Life Sciences, College of Health, Medicine and Life Sciences, Brunel University \\ London, Uxbridge, UK; ${ }^{3}$ Division of Gastroenterology and Hepatology, Medical University of South Carolina, Charleston, SC, USA; ${ }^{4}$ Section of \\ Gastroenterology, Ralph H. Johnson Veterans Affairs Medical Center, Charleston, SC, USA; ${ }^{5}$ Department of Physiology, Faculty of Medicine and \\ Nursing, University of the Basque Country, Universidad del Pais Vasco/Euskal Herriko Unibertsitatea (UPV/EHU), Leioa, Spain \\ Correspondence to: Salvatore Papa. Leeds Institute of Medical Research at St. James', Faculty of Medicine and Health, University of Leeds, St. James' \\ University Hospital, Leeds, UK. Email: s.papa@leeds.ac.uk; Wing-Kin Syn. Division of Gastroenterology and Hepatology, Medical University of \\ South Carolina, Charleston, SC, USA. Email: synw@musc.edu. \\ Comment on: Conde de la Rosa L, Garcia-Ruiz C, Vallejo C, et al. STARD1 promotes NASH-driven HCC by sustaining the generation of bile acids \\ through the alternative mitochondrial pathway. J Hepatol 2021;74:1429-41.
}

Submitted Sep 14, 2021. Accepted for publication Oct 18, 2021.

doi: 10.21037/hbsn-21-374

View this article at: https://dx.doi.org/10.21037/hbsn-21-374

Having cholesterol in our body is essential for living healthy, but too much can lead to serious health problems, including — but not limited to- - heart attacks and strokes (1). Indeed, accumulating evidence points to cholesterol as a cancer risk factor especially in colon, rectal, prostatic and testicular cancer (2). Cholesterol is the most abundant sterol in animal tissues with many important functions that spans from simply maintaining cell membrane physical properties to the production of bile acids and biosynthesis of steroid hormones. For the most part, cholesterol is synthetised de novo in the liver, but some is obtained from dietary intake. The synthesis of cholesterol takes place in the cytoplasm and the endoplasmic reticulum (ER) of hepatocytes (the major liver cell types) and starts with one molecule of acetyl-CoA. Through a series of enzymatic reactions, acetyl-CoA is converted into farnesyl pyrophosphate, which undergoes condensation to form squalene and then the direct precursors of cholesterol, lathosterol and desmosterol $(1,2)$. The liver is also the main site of cholesterol catabolism resulting in the formation of bile acids and oxysterols (oxidized cholesterol derivatives) such as 27-hydroxycholesterol and 25-hydroxycholesterol generated through the enzymatic activity of the cytochrome P450 enzymes, CYP27A1 and CYP3A4 (1). Interesting, recent studies have demonstrated that cholesterol derivatives, including 27-hydroxycholesterol, display tumour promoting properties $(1,3)$. Mice fed with high cholesterol develop nonalcoholic steatohepatitis (NASH) and hepatocellular carcinoma (HCC), the primary form of liver cancer (4). Like other types of cancer cells, HCC cells proliferate at a very high rate thus continuously requiring de novo cell membrane lipids and fatty acid synthesis. However, the underlying mechanisms by which dietary cholesterol contributes to the development of liver cancer is unknown. Interestingly, a recent report from Conde de la Rosa et al. (5) suggested a key role for steroidogenic acute regulatory protein 1 (STARD1) in cholesterol-induced liver cancer (Figure 1). STARD1 is one of the 15 distinct mammalian proteins (STARD1-STARD15) containing a lipid transfer domain spanning $\sim 210$ residues that serves as a binding interface for lipids (6). STARD1 regulates the ratelimiting step in steroid hormone production pathway in response to hormonal stimuli by transferring cholesterol from the outer to the inner mitochondrial membrane, allowing the cholesterol side chain to be cleaved by the $\mathrm{P} 450$ side chain cleavage complex and to generate pregnenolone (6). In the liver, mitochondrial cholesterol is converted to 27-hydroxycholesterol and ultimately into chenodeoxycholic acid (CDCA) through activation of the alternative (acidic) mitochondrial pathway of bile acids biosynthesis $(4,5)$. How STARD1 contributes to HCC pathogenesis is, however, debatable. STARD1 is 


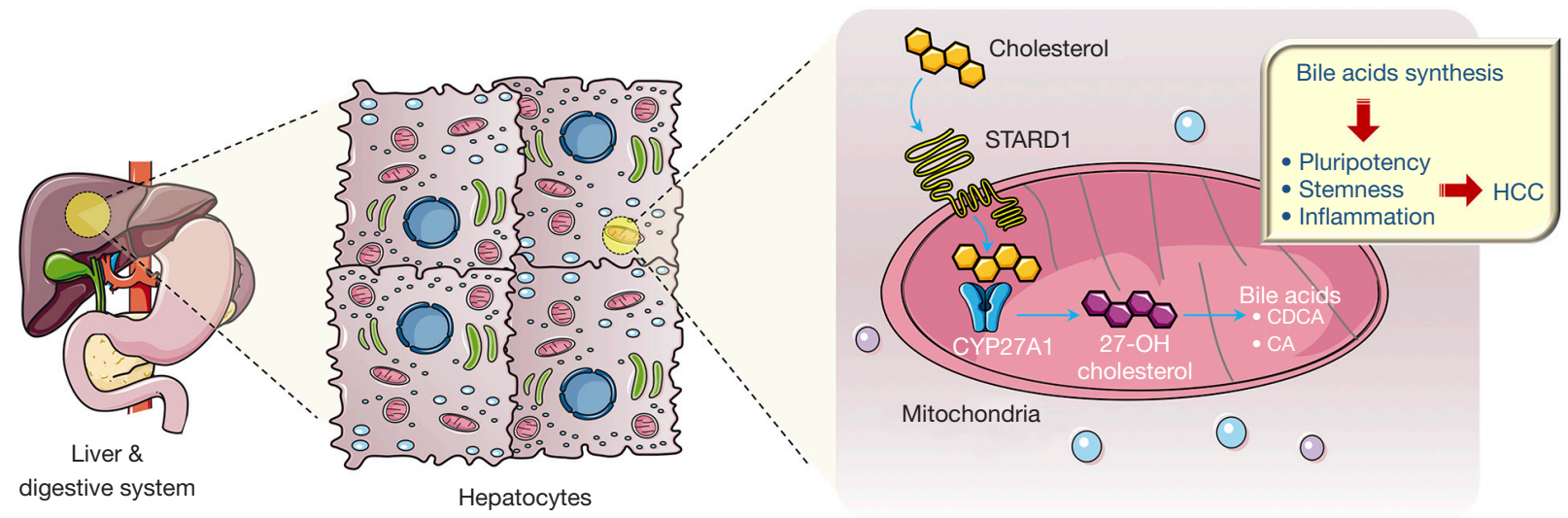

Figure 1 Simplified illustration of STARD1 regulation of cholesterol-driven NASH and HCC. Schematic illustration depicting how mitochondrial cholesterol contributes to the development of NASH-driven HCC. When the intracellular levels of cholesterol reach a certain threshold, it undergoes trafficking to different intracellular organelles, including mitochondria. STARD1 protein mediates cholesterol transport to the mitochondrial inner membrane, supplying cholesterol to CYP11A1 to generate pregnenolone in steroidogenic tissues, or to CYP27A1 to generate oxysterols (i.e., 27-OH cholesterol) in hepatocytes and other cells expressing this enzyme. Oxysterols are then metabolized into bile acids such as CDCA and CA, the two major primary bile acids synthesized in human livers that are conjugated with taurine or glycine for secretion into bile. The amount of bile acids accumulated in the liver are, therefore, strictly reliant on STARD1 expression levels that remain low in a normal functioning liver but increase several folds in response to acute liver injury and chronic liver disease, such as NASH—one of most prevalent causes of HCC. CDCA, chenodeoxycholic acid; CA, cholic acid; HCC, hepatocellular carcinoma; NASH, nonalcoholic steatohepatitis.

highly expressed in the livers of patients with NASHassociated HCC and its expression occurs mainly in hepatocytes and-to a lesser extent-in Kupffer cells and hepatic stellate cells. Conde de la Rosa et al. (5) demonstrated that mice pre-treated with the carcinogen diethylnitrosamine (DEN) and then fed with a high fat diet (HFD) supplemented with high cholesterol (namely HFHC diet) exhibited high levels of STARD1 along with enhanced expression of inflammatory signals (i.e., TNF- $\alpha$ and CCL2) and HCC markers (i.e., AFP and YAP). Importantly, STARD1 gene deletion (STARD1 ${ }^{\Delta \mathrm{Hep}}$ ) in hepatocytes attenuated NASH-driven HCC. This was elegantly shown in two distinct animal models fed with HFHC diet that most closely resembles human NASHdriven HCC: (I) the MUP-uPA Tg model where mice express transiently high amounts of urokinase plasminogen activator (UPA) in hepatocytes resulting in hepatocyte ER stress and transient liver damage (events implicated in human NASH development); (II) DEN carcinogen model where mice develop HCC after DEN injection at 15 days post-birth. In addition to using STARD $1^{\Delta H e p}$ mice to test the effect of STARD1 genetic deletion, the authors used adenoviral-induced overexpression of STARD1 (STARD1 ${ }^{\mathrm{OE}}$ ) in mouse livers to test the effect of
STARD1 overexpression in response to DEN + HFHC diet treatment (5). As anticipated, STARD1 overexpression in hepatocytes was associated with an increase in inflammatory signals and tumour formation compared to mouse livers expressing endogenous STARD1. Strikingly, increased HCC formation was evident only in STARD $1^{\mathrm{OE}}$ mice fed with $\mathrm{HFHC}$ but not in STARD $1^{\mathrm{OE}}$ mice fed with a regular diet, thus supporting the notion that high cholesterol is required to prime NASH-driven HCC (5). By comparing gene and protein expression in livers of STARD $1^{\text {AHep }}$, MUP$\mathrm{uPA} / \mathrm{STARD} 1^{\triangle \mathrm{Hep}}$ and STARD $1^{\mathrm{OE}}$ mice and respective controls, Conde de la Rosa et al. (5) further demonstrated that levels of STARD1 in HCC positively correlated with an increase in signalling pathways related to bile acid synthesis, and in particular, unconjugated bile acids, such as cholic acid (CA) and $\beta$-muricholic acid ( $\beta$-MCA), and their tauro-conjugated derivatives (Figure 1). The authors also found that overexpression of STARD1 was associated with elevated levels of genes involved in cell self-renewal and pluripotency, suggesting a role for STARD1 in regulating stemness in HCC (Figure 1). Interestingly, no correlation of expression was found with farnesoid $\mathrm{X}$ receptor (FXR), a bile acid ligand-activated transcription factor that is involved in bile acids synthesis. These data suggest 
that STARD1 regulation of bile acids synthesis during development of NASH-driven HCC is independent of FXR. In conclusion, Conde de la Rosa et al. (5) unravelled a key role for STARD1 in the synthesis of bile acids during the development of NASH-HCC (Figure 1). The question that remains to be addressed is how levels of STARD1 are regulated in human and mouse NASH-HCC. Given that the expression of STARD1 in response to acetaminophen (APAP)-induced liver injury was found to be dependent on c-Jun N-terminal kinase (JNK) (7), a stress activated protein kinase implicated in liver homeostasis and cancer (8-10), it would be worth to evaluate whether expression of STARD1 is regulated by JNK or its downstream effectors (8) in NASH and HCC. Further studies are also needed to determine whether STARD1 is a potentially targetable protein for the treatment of patients with NASH-HCC.

\section{Acknowledgments}

Figures were created modifying illustrations provided by Servier (https://smart.servier.com) under Creative Commons Attribution 3.0 Unported License.

Funding: SP and CB acknowledge the research funding from Rosetrees Trust (M894) and Guts UK (DGO2019_02).

\section{Footnote}

Provenance and Peer Review: This article was commissioned by the editorial office, Hepatobiliary Surgery and Nutrition. The article did not undergo external peer review.

Conflicts of Interest: All authors have completed the ICMJE uniform disclosure form (available at https://hbsn. amegroups.com/article/view/10.21037/hbsn-21-374/coif). The authors have no conflicts of interest to declare.

Ethical Statement: The authors are accountable for all aspects of the work in ensuring that questions related to the accuracy or integrity of any part of the work are appropriately investigated and resolved.

Open Access Statement: This is an Open Access article distributed in accordance with the Creative Commons Attribution-NonCommercial-NoDerivs 4.0 International License (CC BY-NC-ND 4.0), which permits the noncommercial replication and distribution of the article with the strict proviso that no changes or edits are made and the original work is properly cited (including links to both the formal publication through the relevant DOI and the license). See: https://creativecommons.org/licenses/by-nc-nd/4.0/.

\section{References}

1. Tabas I. Cholesterol in health and disease. J Clin Invest 2002;110:583-90.

2. Giacomini I, Gianfanti F, Desbats MA, et al. Cholesterol Metabolic Reprogramming in Cancer and Its Pharmacological Modulation as Therapeutic Strategy. Front Oncol 2021;11:682911.

3. Voisin M, de Medina P, Mallinger A, et al. Identification of a tumor-promoter cholesterol metabolite in human breast cancers acting through the glucocorticoid receptor. Proc Natl Acad Sci U S A 2017;114:E9346-55.

4. Liang JQ, Teoh N, Xu L, et al. Dietary cholesterol promotes steatohepatitis related hepatocellular carcinoma through dysregulated metabolism and calcium signaling. Nat Commun 2018;9:4490.

5. Conde de la Rosa L, Garcia-Ruiz C, Vallejo C, et al. STARD1 promotes NASH-driven HCC by sustaining the generation of bile acids through the alternative mitochondrial pathway. J Hepatol 2021;74:1429-41.

6. Alpy F, Tomasetto C. Give lipids a START: the StARrelated lipid transfer (START) domain in mammals. J Cell Sci 2005;118:2791-801.

7. Torres S, Baulies A, Insausti-Urkia N, et al. Endoplasmic Reticulum Stress-Induced Upregulation of STARD1 Promotes Acetaminophen-Induced Acute Liver Failure. Gastroenterology 2019;157:552-68.

8. Lepore A, Choy PM, Lee NCW, et al. Phosphorylation and Stabilization of PIN1 by JNK Promote Intrahepatic Cholangiocarcinoma Growth. Hepatology 2021;74:2561-79.

9. Manieri E, Folgueira C, Rodríguez ME, et al. JNKmediated disruption of bile acid homeostasis promotes intrahepatic cholangiocarcinoma. Proc Natl Acad Sci U S A 2020;117:16492-9.

10. Cubero FJ, Mohamed MR, Woitok MM, et al. Loss of c-Jun $\mathrm{N}$-terminal Kinase 1 and 2 Function in Liver Epithelial Cells Triggers Biliary Hyperproliferation Resembling Cholangiocarcinoma. Hepatol Commun 2020;4:834-51.

Cite this article as: Papa S, Bubici C, Syn WK. STARD1: a new rising StAR in cholesterol-mediated hepatocarcinogenesis. HepatoBiliary Surg Nutr 2021;10(6):910-912. doi: 10.21037/ hbsn-21-374 